\title{
Highest efficiency and ultra low emission - internal combustion engine 4.0
}

In the future, the simultaneous reduction of pollutant and $\mathrm{CO}_{2}$ emissions will require significantly enhanced powertrain functionalities that cannot only be adequately represented by the ICE (internal combustion engine) alone. Both automated transmissions and especially powertrain electrification can help to meet efficiently those extended requirements. The extended functionalities are no longer applied exclusively with the ICE itself ("Fully Flexible Internal Combustion Engine"), but distributed across the entire powertrain ("Fully Flexible Powertrain"). In addition, the powertrain will be fully networked with the vehicle environment and thus will utilize all data that are useful for emission and consumption-optimized operation of the ICE.

Combustion engine and electrification often complement each other in a synergetic way. This makes it extremely sensible for the combustion engine to evolve in future from a "single fighter" to a "team player". If one compares the requirements of such an ICE with the definition of Industry 4.0, then there are extensive correspondences. Thus, it seems quite opportune to call such a fully networked combustion engine designed to meet future needs as "Internal Combustion Engine 4.0 (ICE 4.0)". This even more so, as such a name can also be derived from the history: e.g. ICE 1.0 describes the combustion engines of the first mass-produced vehicles, ICE 2.0 the combustion engines emission-optimized since the 1960s and ICE 3.0 the highly optimized "Fully Flexible Combustion Engine", which currently offers a high torque and performance potential combined with low fuel consumption and pollutant emissions.

In addition to further improvements in fuel consumption, the "Combustion Engine 4.0" offers such a low level of pollutant emissions that can best be described as "Zero Impact Emission". This means that such future ICE's will no longer have a negative impact on the imission situation in urban areas. With the e-fuels topic, the ICE also has the potential to become both $\mathrm{CO}_{2}$ - and pollutant-neutral in the medium and long term. This means that the ICE - also in passenger cars - will continue to be an essential and necessary cornerstone for future powertrain portfolios for the next decades.

Key words: passenger car, gasoline, diesel, hybrid, powertrain

\section{Introduction}

The combustion engine is currently undergoing the most turbulent phase in its more than 100-year of history. From the undeniable enabler of individual mobility, the ICE is partly driven into a negative image as an air polluter and no longer being a future oriented technology. Particularly in Europe, the combination of exceeding imission limits in major cities with the emissions issue of the Diesel engine, has had a lasting impact on the image of the ICE. Potential city access restrictions not only generate anxiety scenarios, but also increasingly determine the buying behavior of the customer and thus, not at least, question the future of the Diesel engine in passenger cars. In the public discussion, however, most often neither the resulting losses in people's assets and the contribution of non-automotive pollutant emitters to the imission situation are considered.

Looking at the challenges of the ICE in a technically rational way against a global background, it is probably less the issue of pollutants, but rather the sustainable reduction of $\mathrm{CO}_{2}$ emissions, that presents the biggest challenge, Fig. 1.

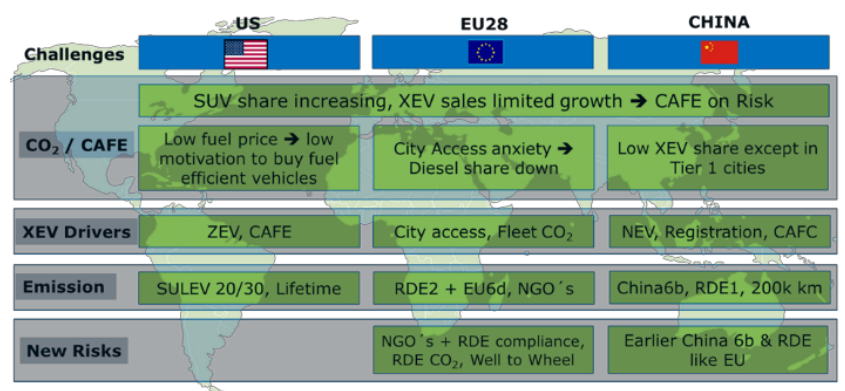

Fig. 1. Global and regional challenges for the ICE
The fulfillment of the worldwide $\mathrm{CO}_{2}$ resp. fuel consumption fleet limits is hampered both by a strongly increasing portion of vehicles with high weight and driving resistance (SUV boom) and thus unfavorable consumption behavior compared to the passenger car as well as a still restrained purchase behavior for XEV's.

In the US, low fuel prices give the customer a low economic motivation to buy $\mathrm{CO}_{2}$-efficient vehicles. In addition, since the US legislation is traditionally more pollutantoriented than emissions-oriented, a more modest transition to electromobility is expected.

In Europe, on the other hand, demanding $\mathrm{CO}_{2}$ reductions in conjunction with declining Diesel and SUV shares, requires a relatively rapid expansion of electrification just to meet the $\mathrm{CO}_{2}$ fleet targets. In addition, the shift towards electrification is enhanced additionally by the ongoing discussions about bans for the ICE.

In China, above all, the imission situation in mega cities and the resulting registration restrictions and exemptions for new energy vehicles (NEVs) are a major motivation for electric drives.

Although the United States, especially California, have been at the forefront of low pollutant emission legislation for decades, in future both China with its extremely low thresholds in the test cycle (China $6 \mathrm{~b}$ ) and demanding durability requirements, and especially Europe with a very challenging RDE legislation will become the pacemakers.

The short- and medium-term technology trends can be estimated in a first approximation from the already apparent or expected legislation, where strong regional differences are to be expected. Long-term trends, on the other hand, are mainly due to the $\mathrm{CO}_{2}$ reductions required to meet the cli- 
mate goals. However, these must be seen as balance between the $\mathrm{CO}_{2}$ emissions of the transport sector ("Tank- toWheel") and the provision of primary energy ("Well-toTank"). Although the division into individual sectors allows the definition of separate target values, there is the risk that over the time course the overall optimum of $\mathrm{CO}_{2}$ reduction will not always be achieved. For example, a reduction of purely vehicle-related tank-to-wheel emissions by electric vehicles only becomes effective in the total $\mathrm{CO}_{2}$ emissions, if the well-to-tank emissions of the energy supply are reduced beforehand.

Of course, in addition to technical progress in development, a wealth of legal and political influencing factors determines the long-term development of technology. The issues of registration and access restrictions ("Diesel-Ban", "ICE-Stop") certainly play a decisive role, Fig. 2.

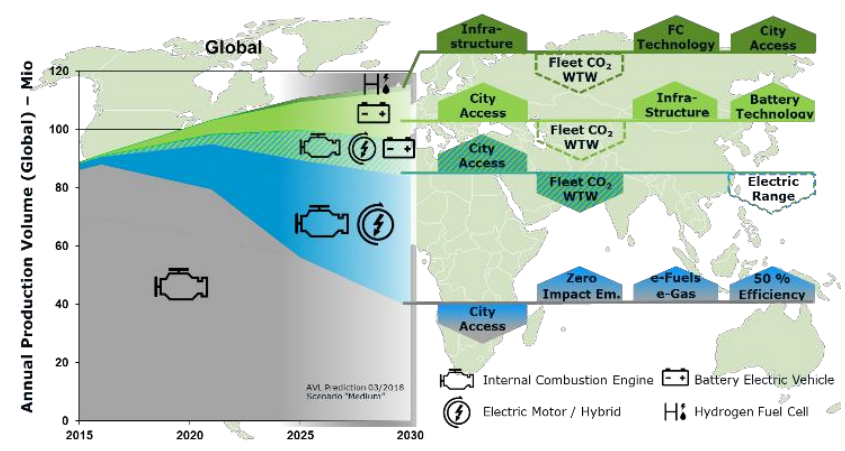

Fig. 2. Main factors influencing the long-term global technology distribution [1]

In addition to the reduction of pollutant emissions, fulfilling the fleet $\mathrm{CO}_{2}$ limits is a key driver for electric vehicles, whose emission contribution is currently rated "zero". Thus, a transition from a tank-to-wheel to a well-to-wheel approach with today's powerplant $\mathrm{CO}_{2}$ footprint would significantly reduce the lever of the electric vehicles in fleet $\mathrm{CO}_{2}$ and, above all, reduce the attractiveness of plug-in hybrids (PHEV). Similarly, advances in battery technologies are also competing the need for PHEV as a long-haul solution, even though the general fast-charging issue of increasing inventory of BEVs with very high battery capacity in long-haul operations is challenging.

The success of the fuel cell is much more determined by the infrastructure than in the case of the battery-powered vehicle. Here, in a global view, both the storage and the distribution of hydrogen are still inhibitions. However, vehicle refueling itself can be solved in a practical way by the possible transfer of high energy contents within a brief time.

With the ICE, on the other hand, the topics "Zero Impact Emission" and "e-Fuels" as well as "e-Gas" also make it possible to operate the ICE practically pollutant-free and $\mathrm{CO}_{2}$-neutral, thus bringing it to a level with renewable sources in terms of environmental impact in a then fair competition again.

\section{A new generation of ICE's}

Basically, there are two options for meeting future $\mathrm{CO}_{2}$ fleet targets and emission limits:
- Electrification focus: The emphasis is placed on the highest possible proportion of electrification (BEV + PHEV), if necessary, a corresponding market penetration is promoted with price support measures. In the case of conventional drives, the focus is primarily only on the adaptation to the legal boundaries, most of the $\mathrm{CO}_{2}$ fleet reduction is represented by BEV and PHEV.

- Balanced approach: In addition to the "marketable" growth of $\mathrm{BEV}+\mathrm{PHEV}$, the further reduction of $\mathrm{CO}_{2}$ emissions is the result of a significant advancement of ICE based powertrains utilizing synergies with affordable electrification measures.

However, looking at the current evolution of $\mathrm{CO}_{2}$ fleet levels in Europe - the progression of $\mathrm{CO}_{2}$ reduction is well above the target corridor for meeting the fleet targets for 2020 due to rising SUV and falling diesel emissions - all technical options for $\mathrm{CO}_{2}$ reduction will have to be exploited. In conjunction with the also tightened RDE requirements, this requires a sustainable improvement or new development of ICE based powertrains.

Since the issue of real world emissions will increasingly come into the focus, it is becoming more sensible to increasingly use the traffic and environmental information available through the networking of the automobile to optimize fuel consumption and pollutant emission of the powertrain. To use such a potential, it is necessary to integrate electrical functionalities into the powertrain in addition to refined ICE attributes.

If one compares these requirements to future powertrains with the definition of Industry 4.0, in Germany, then surprising matches are found:

Definition Industry 4.0 [2] (in extracts, in the following in italics):

- "Industrial production should be integrated with modern information and communication technology". This also applies also to automobile and optimized powertrain management.

- "The technical basis for this are intelligent and digitally networked systems. With their help, a largely selforganized production is to be possible: people, machines, equipment, logistics and products communicate and cooperate directly with each other in Industry 4.0". This sentence describes in an analogous manner also autonomous driving functions and the networking of the automobile.

- "Networking should make it possible to optimize not just a single production step but an entire value chain". Also in the automobile, it will be possible in the future, to optimize not only the driving itself, but the entire transport process including energy-optimal route selection, maximizing the recuperation potentials and automation of assistance functions such as automatic parking.

- "The network should also encompass all phases of the product's lifecycle - from the idea of a product to development, manufacturing, use and maintenance to recycling." In the future, the evaluation of the automobile and the various drives will also be increasingly carried out on a "lifecycle" basis involving production, operation and recycling.

Thus, it is perfectly opportune to call an ICE designed comprehensively for future needs as "Internal Combustion 
Engine 4.0 (ICE 4.0)". But also from the history can be derived such a name, Fig. 3.

"ICE 1.0" describes the combustion engines of the first mass-produced vehicles. Affordability and reliability were the defining parameters.

In California, the first emission limits for motor vehicles were already set in the 1960s, which is certainly a decisive milestone, e.g. defined as "ICE 2.0".

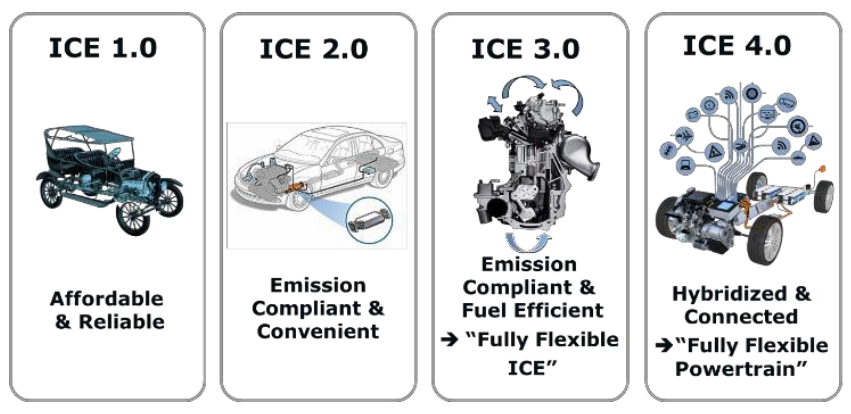

Fig. 3. Generations of ICE based passenger car power- train systems

In the following decades, the limit values for pollutant emissions and fleet consumption target values to be proven on the chassis dynamometer were steadily tightened and required significantly increased variability, the "Fully Flexible Internal Combustion Engine - here described as "ICE 3.0".

The future requirements regarding pollutant as well as $\mathrm{CO}_{2}$ emissions, require significantly extended functionalities of the powertrain, which no longer can be mapped exclusively within the ICE ("Fully Flexible Combustion Engine") but must be distributed to the entire drive train ("Fully Flexible Powertrain"). In addition, the drivetrain will be networked with the vehicle environment, enabling all data useful for emission-optimal powertrain operation to be utilized, and will increasingly be powered by $\mathrm{CO}_{2}$ neutral fuels from renewable sources - "ICE 4.0".

\section{Zero Impact Emission}

In addition to the currently negatively biased image of the ICE, the complex discussions regarding access restrictions for emission-critical zones are causing uncertainty among the buyer. Especially the lack of clearly defined future framework conditions becomes an existential threat to the Diesel engine in passenger cars already short term.

In the medium and long term, a general sales stop of ICE is increasingly being discussed, without considering the untapped potential of future ICE-based powertrain systems. To remain accepted, at least in the European public opinion, it is generally necessary for the ICE to lower its pollutant emissions to a level that is no longer relevant for the environment. With EU6dtemp incl. RDE, a decisive step has already been made in this direction. It can be assumed that such vehicles will certainly fall below the imission limit values in today's imission-critical zones.

"Zero Impact Emission" means that emissions are so low that they have no impact on air quality. To assess this, Fig. 4 shows the emission characteristics of a standard EU6dtemp vehicle with a supercharged DI Gasoline engine in the WLTP cycle.

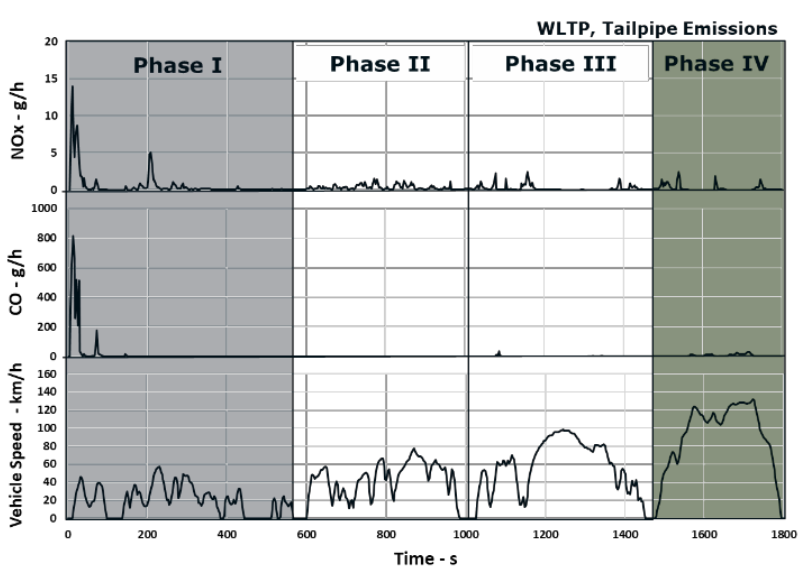

Fig. 4. Pollutant emission of an EU6dtemp vehicle with Gasoline engine in the WLTP

With the Gasoline engine, a strong concentration of pollutant emissions on the non-warmed up or highly dynamic engine operation is obvious. These relations are not only valid for this single vehicle, but are also confirmed on a broad statistical basis, Fig. 5 .

If one looks at the pollutant emissions in warmed up condition, then an emission level of only $2-5 \mathrm{mg} / \mathrm{km}$ is observed, which is an order of magnitude lower then under cold conditions. If a model simulation of critical imission measurement points for Germany - e.g. the Neckartor in Stuttgart [3] - is done then it can be seen that the vehicle emissions only contribute by $0.2-0.5 \mu \mathrm{g} / \mathrm{m}^{3}$. This is uncritical compared to the legal limit $\left(40 \mu \mathrm{g} / \mathrm{m}^{3}\right)$ as well as compared to the emissions of the non-automotive sector (approx. $18 \mu \mathrm{g} / \mathrm{m}^{3}$ ). So for warm engine EU6d temp vehicles could already be called „Zero Impact Emission“.

Starting from the EU6d temp status, the main challenge for further emission reductions are with the improvement of pollutant emissions at ICE start and in non-warmed up operating range, as well as in the temperature management of the exhaust gas aftertreatment. In addition, various measures to limit pollutant emissions are required in the upper load range.
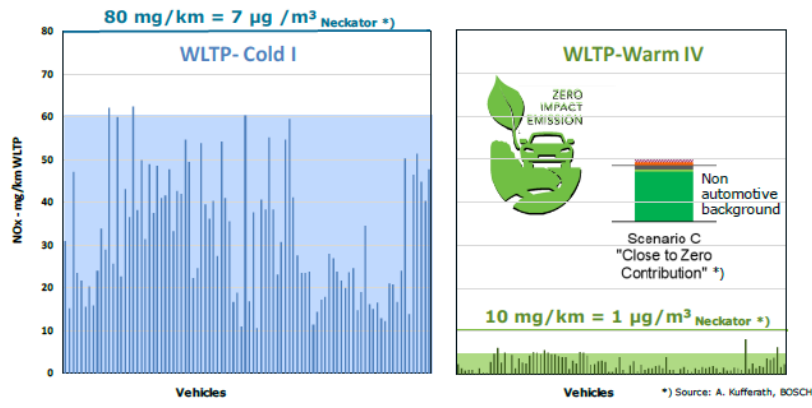

Fig. 5. Emission behavior of EU6dtemp vehicles with Gasoline engines

For both concern areas, it makes sense to utilize synergy effects with mild hybridization. If you set e. g. a suitably designed 48V system to make the cold operation primarily electrically and at the same time to condition the exhaust aftertreatment by means of catalyst heating, the cold emissions can be significantly reduced. Since this mild hybridization also dampens emission-relevant dynamic peaks even 
when warm (limitation of the ICE dynamics), it is possible to achieve an emission level that justifies talking about "Zero Impact Emission".

\section{Electrification of the ICE}

In the future, the simultaneous reduction of both pollutant and $\mathrm{CO}_{2}$ emissions will require significantly enhanced functionalities of the powertrain, which can only be insufficiently represented by the ICE alone. Both automated transmissions and electrification can help to efficiently cover the extended functionalities. These functionalities are no longer exclusively applied within the ICE itself ("Fully Flexible Internal Combustion Engine"), but distributed across the entire powertrain ("Fully Flexible Powertrain"), Fig. 6.

Clever balancing of the complementary properties of electrification and ICE results in a synergetic potential for improvement in terms of reducing both pollutant and $\mathrm{CO}_{2}$ emissions. Thus, in future the ICE will change "from a lone fighter to a team player".

As a result, especially with a high degree of electrification, a simplification of the ICE will be also possible. If e.g. the lowest load range of the ICE is replaced by purely electric driving or load shift by charging the battery, the layout of the ICE can focus on the area of best specific fuel consumption respectively low specific pollutant emissions (with Gasoline engine e.g. layout of the turbocharger for higher mass flow, possibly omission of variable valve lift, modified layout of variable compression, etc.).

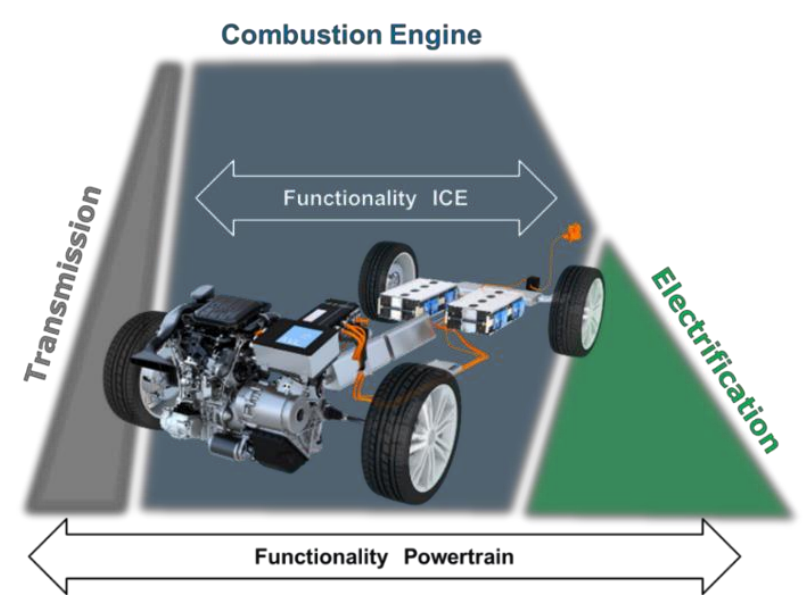

Fig. 6. From "Fully Flexible Combustion Engine" to "Fully Flexible Powertrain" [1]

Already a sufficiently graded automated transmission with a large spread helps to operate the ICE in most of the driving situations in the respectively optimum map area usually best specific fuel consumption ("Sweet Spot Follower" - yellow area in Fig. 7). With appropriate hybridization, even at low power demand the load point can be shifted towards a high-efficiency range either by electrical recuperation and charging of the battery or, in the case of sufficient battery charge, driven purely electrically (lower green area in Fig. 7).

In the upper load range, of course, the electrical torque can be used to increase the total torque (dashed green line). Regarding emission and fuel consumption reduction, how- ever, it is more sensible to reduce the torque and dynamic demand of the ICE by means of the additional electrical torque at least partially, especially with low engine speeds (upper green area in Fig. 7). This not only allows avoidance of operating ranges with unfavorable performance of the ICE, but also additional synergy effects e.g. a modified design of the turbocharger better matched to the higher flow range (expansion of the stoichiometric operating range). In view of the expected changes in RDE legislation [4], such a "dynamic limitation" of the ICE also gains additional importance for a robust RDE calibration. However, the electrical torque is only temporary, according to the electric energy available, which must be considered in the system design accordingly.

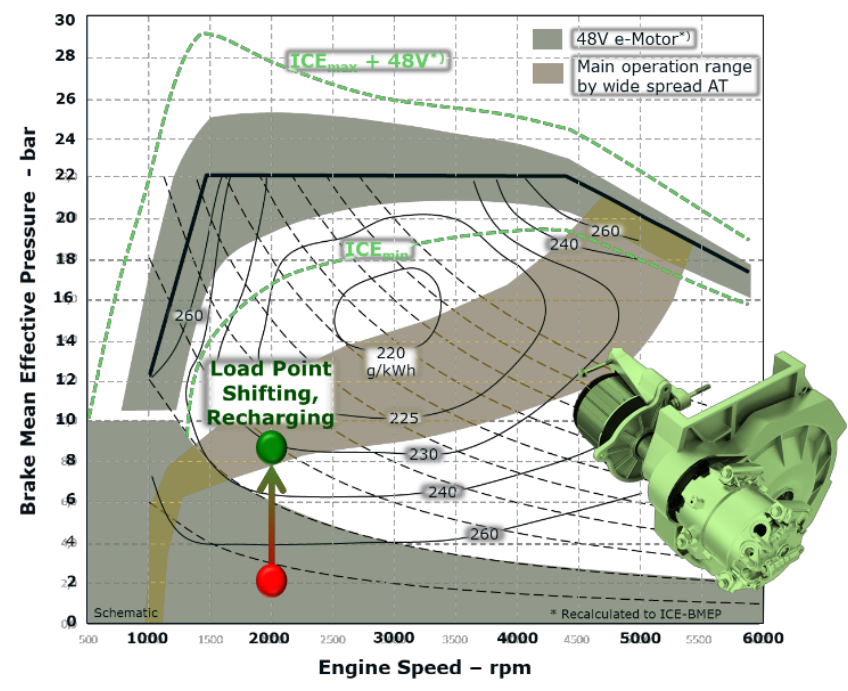

Fig. 7. Interaction of ICE, Automated Transmission and Mild Hybridization $(48 \mathrm{~V})$

Depending on the respective ICE, transmission and electrification strategy, different arrangements of the electric motor prove to be the respectively most favorable variant, Fig. 8. Both modular and dedicated solutions are used $[5,6]$.

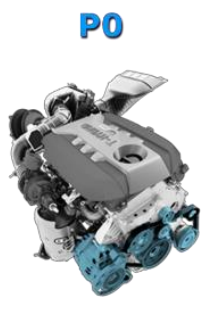

P2

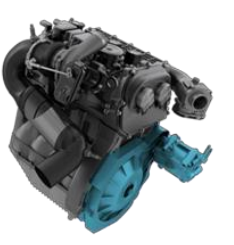

P3

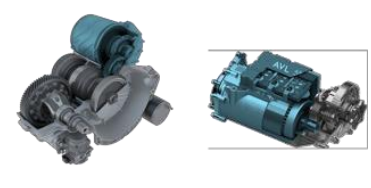

Fig. 8. Electrification of ICE-based powertrain systems

\section{Development of the Gasoline Engine}

\subsection{Challenges}

Due to its in stoichiometric operation highly efficient exhaust aftertreatment, the Gasoline engine has so far been much less in the center of public emission discussions than the Diesel engine. However, the new RDE legislation, especially the tightening of Package 3 and 4, represents a huge challenge. Thus, e.g. the dynamic limitation introduced to restrict aggressive driving allows, to a certain 
extent, even extreme driving maneuvers such as full load drive-off with cold engine. Consequently, reducing the dynamics of the cold drive-off becomes a crucial criterion for safely meeting future RDE requirements.

The strongest temperature and dynamic influence is obtained in the gasoline engine for the number of particles, Fig. 9.

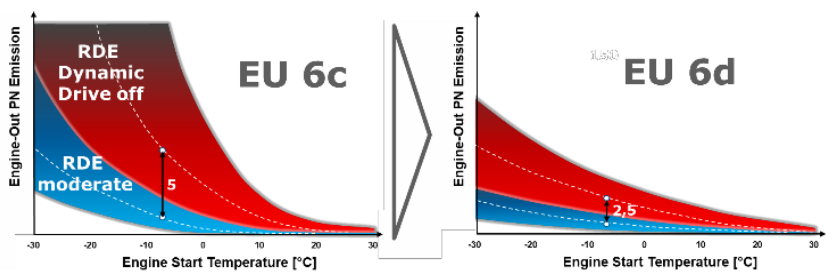

Fig. 9. Influence of start temperature and driving style on the engine-out particulate emissions in the RDE city section - TGDI engines

Since particulate filters for Gasoline engines, at least in the new or freshly regenerated state have significantly lower filtration efficiencies than Diesel particulate filters, a reduction of the particle engine out emissions is still required despite the broad introduction of the particulate filter also with Gasoline engine. By detail optimization both the temperature and the dynamic sensitivity of PN generation could be significantly improved from an EU6c status, Fig. 10 left, towards an EU6d status, Fig. 9 right.

Compared to the Diesel engine, the Gasoline engine has a much higher sensitivity to higher exhaust gas back pressure, which gains in importance through the future requirement for stoichiometric operation in the entire map. Thus, the trade-off between filtration efficiency and exhaust back pressure of the GPF becomes a defining parameter for the Gasoline engine. Fig. 10 shows an overview of different filter types with respective filter soot loadings of 0,1 and $3 \mathrm{~g} / \mathrm{l}$.

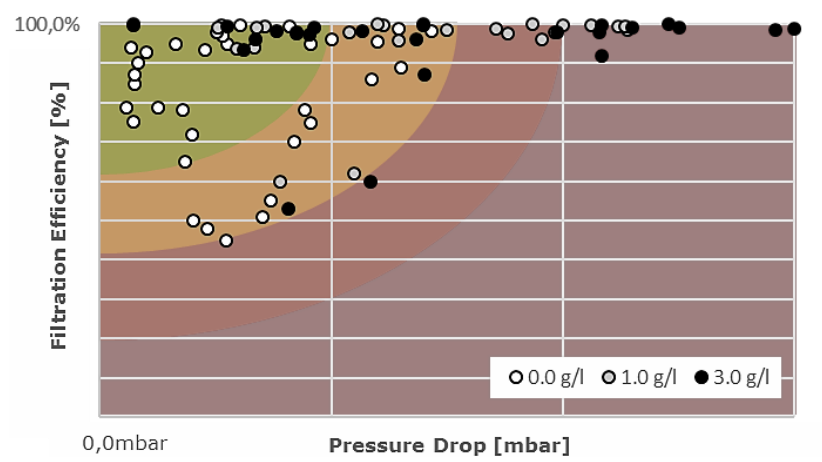

Fig. 10. Trade-off between filter efficiency and exhaust back pressure with different soot loadings

While in the past, high filtration efficiencies were almost inevitably associated with high backpressure (red and yellow areas in Fig. 10), current developments are already leading to much more favorable relations - green area in Fig. 10 - although partially with compromises on filter size. For gas-powered SI-engines this filter problem is eliminated, as from combustion side more or less no particle emission is generated thus increasing the attractiveness of Gas engines additionally.
Finally, the elimination of scavenging strategies necessary to safely comply with $\mathrm{RDE}-\mathrm{NO}_{\mathrm{x}}$, requires appropriate compensation in torque and low-speed response, which in turn argues for a "cooperation" between ICE and powertrain electrification.

In general, some measures for the robust RDE fulfillment are in trade-off with the $\mathrm{CO}_{2}$ emission, the central future development focus of the Gasoline engine. This increases the motivation to use low-cost electrification to reduce $\mathrm{CO}_{2}$ emissions and to improve existing trade-offs, for example with a different layout of the turbomachinery. Since such mild hybridizations can also improve the robustness of emissions reduction in real world operation at the same time, such systems will form a broad basis for future ICE based powertrains.

\subsection{Technology approaches Gasoline engine}

The current technical mainstream of Gasoline engines extended expansion by Miller/Atkinson cycle concepts will continue in the foreseeable future, but will be further optimized by improved charging systems. In addition to this mainstream technology, however, a certain amount of technology diversification will remain. Variable Compression Ratio VCR, Spark Initiated HCCI, advanced ignition and Ultra High Pressure Injection UHP are additional focus points, Fig.11.

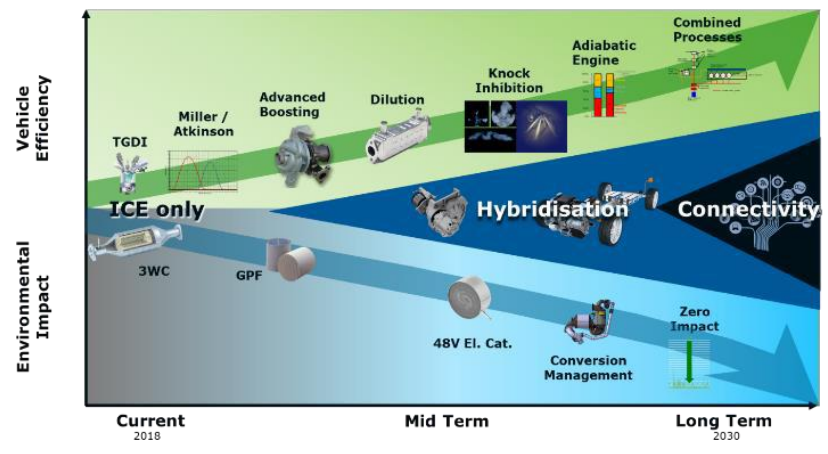

Fig. 11. Technology roadmap Gasoline engine

Once knock can be limited or eliminated isolation of the combustion chamber ("adiabatic engine") can shift energy from the low temperature to the high temperature level. In combined processes these additional energy amounts can be used. Peak efficiency levels of $45 \%$ up to $50 \%$ can be expected in the future.

Future emission requirements such as lowest particulate emissions or stoichiometric operation in the complete engine map require advanced emission concepts beyond the three way catalyst and the particle filter.

By using an electrically heated catalyst with extended preheating/post heating functionality ("conversion management") as well as by hybridization and connectivity further significant emission reduction up to "Zero Impact Emission" level is possible even for not fully warmed up engines.

Synergy effects with hybridization allow an even more fuel consumption-oriented design of the IC, especially of the charging and combustion processes. Since in future, the ICE must increasingly cover various levels of electrifica- 
tion, an expanded modularization will gain an increasing importance even within the ICE. While in the past, different torque and power levels of the ICE were usually covered with different geometric displacements, the topic of charging enables a power and torque spread without changing the engine geometric displacement. Miller and Atkinson cycle allow a separation of geometric and effective compression ratio. Thus, a widely spread power graduation can be displayed at the same geometric displacement within one engine family. The performance differentiation is primarily based on the extent of "Millerization", the boost pressure, the valve lift curves and the geometric compression ratio, Fig. 12.
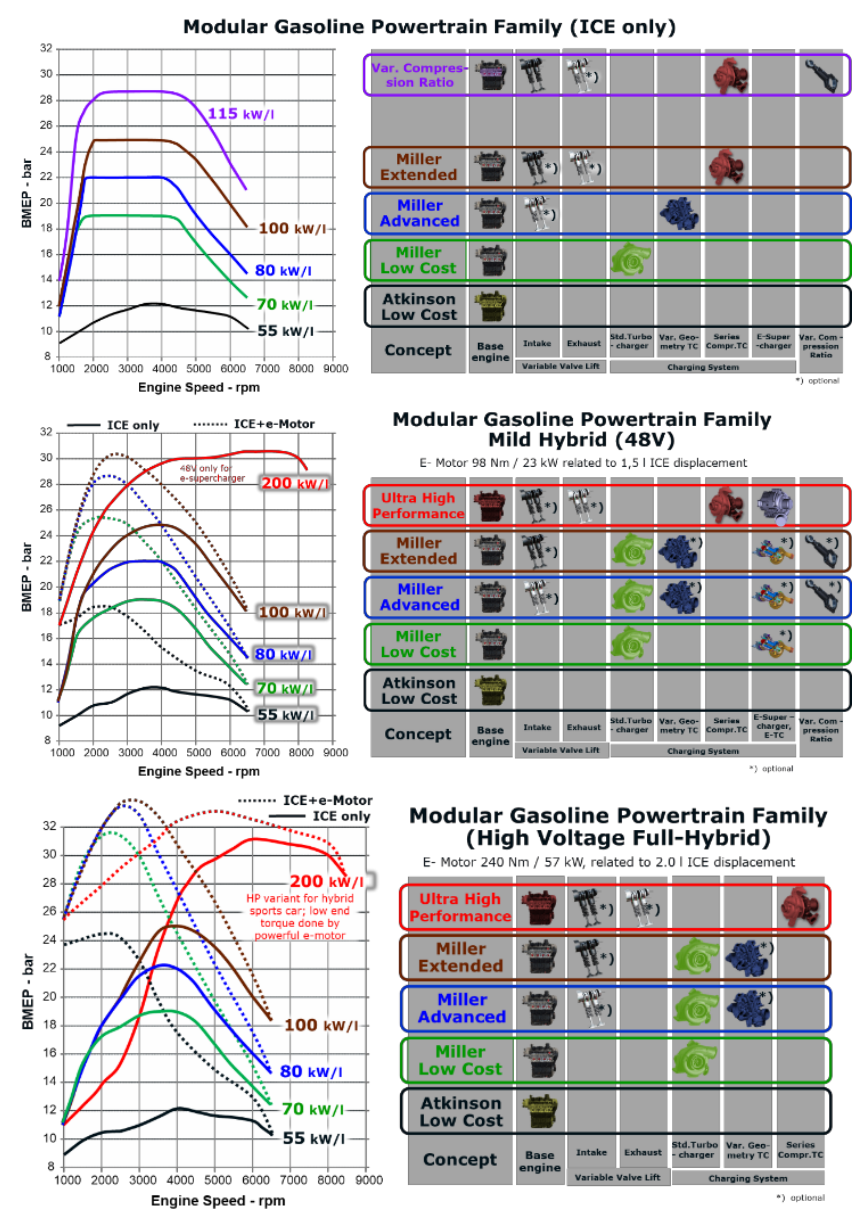

Fig. 12. Modular Gasoline engine concept with different electrification levels

While high torque at low engine speeds is a prerequisite for a fuel economy oriented powertrain layout, it usually results in some compromises with the ICE layout. Thus, an electrical torque assistance at low speeds allows a much more focused orientation of the ICE to the main operating range, Fig. 12 center. In most cases, the charging unit can also be better adapted and if necessary also simplified and the geometric compression ratio can be raised further.

Recovery of exhaust gas energy, for example by means of electrified turbochargers (e-turbo) in combination with the electrified powertrain, is a sensible addition to a further increase in efficiency.
Niche applications with extremely high specific power are made possible by using additional electrical charging. With the increased electrical power of high-voltage full hybrids, this alignment can be taken a step further, Fig.12 lower part. Since considerably higher electrical energies are converted here, an intelligent, preferably predictive energy management is required to assure reproducibility of the acceleration behavior with limited battery capacity.

In contrast to the low speed range, the direct influence of electrification in the rated power range is limited by economically justifiable battery sizes (exception: Plug In Hybrid). However, synergy effects - such as e.g. reduced exhaust back pressure can be used by modified layout of the gas exchange process respectively the turbocharger.

Nevertheless, future legal requirements such as stoichiometric operation in the entire map remain challenging and require either a compromise with $\mathrm{CO}_{2}$ measures or additional expenses. A variety of measures is - either individually or in combination - is used to reduce the exhaust gas temperature so far that an enrichment for component protection can be avoided. Fig. 13 shows an overview of the stoichiometric power potential of the individual technologies and the cross-influence on the minimum specific fuel consumption.

Active exhaust gas cooling in the form of an integrated exhaust manifold is increasingly becoming the basic technology of turbocharged Gasoline engines, but is limited towards higher performance by the high heat input into the cooling system. Miller timing (early inlet valve closure) provides a broad basis for the implementation of high geometric compression ratio and extended expansion and is primarily used to reduce fuel consumption. Improved gas exchange (Variable Turbine Geometry, "Series Compressor Turbocharger" with intercooler) enables lower charge temperatures and better charging efficiencies. Cooled exhaust gas recirculation improves both the stoichiometric power range and the minimum fuel consumption, but at the same time increases the heat input into the cooling system. Variable geometric compression ratio, especially in conjunction with variable intake valve lift proves to be very effective both in view of stoichiometric power and fuel consumption. And finally, water injection - albeit with respective water consumption - enables stoichiometric operation up to more than $170 \mathrm{~kW} / \mathrm{l}$.

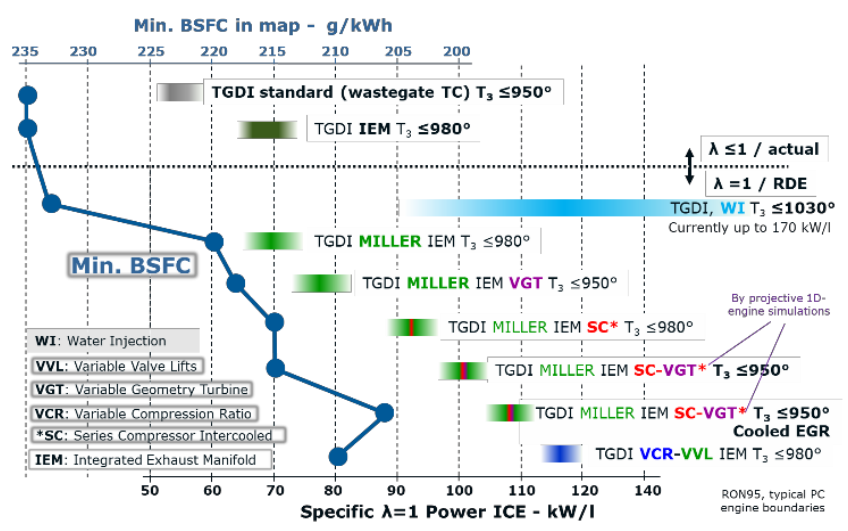

Fig. 13. Measures to increase the stoichiometric power range, RON 95 
With increasing electrification, the focus of fuel consumption is shifting from the low load range (for example $2000 \mathrm{rpm}, 2.0$ bar BMEP) towards higher engine loads to the area of minimum specific fuel consumption. Thus, the ratio of maximum efficiency to maximum specific power becomes a decisive engine parameter, Fig. 14.

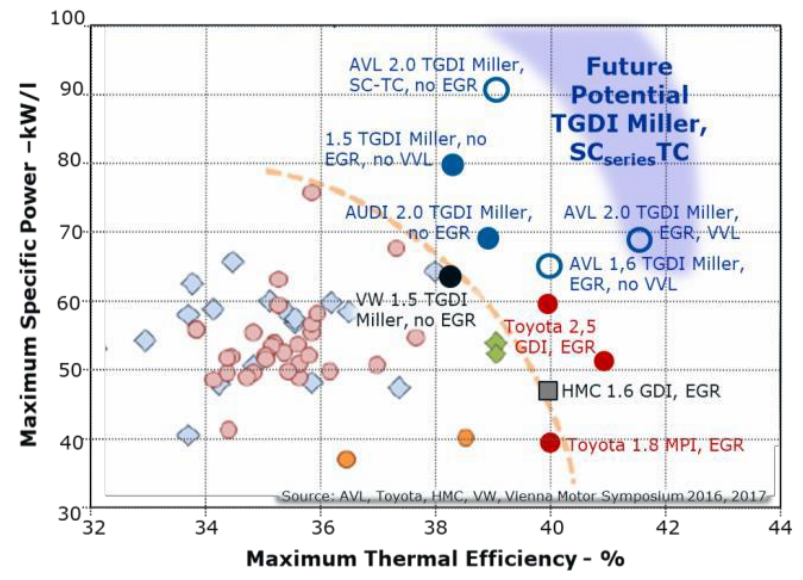

Fig. 14. Trade-off between max. efficiency and max. specific performance

Looking at the state of the art, Miller/Atkinson concepts represent the most attractive low-consumption solutions. With advanced charging systems and refined combustion processes, a significant shift of this trade-off is expected in the future, especially towards higher specific performance. However, the requirements of stoichiometric full load operation will result in increased differentiation in these specific performance levels. Although the concept engines (hollow circles in Fig. 14) are already designed for stoichiometric operation, stoichiometric full load is only applied to a few series engines yet.

While overall efficiencies of up to $45 \%$ appear feasible in the medium term for current developments, in the research stage total efficiencies of more than $50 \%$ are conceivable in the long term with complex processes. This significantly extends the trade-off between maximum efficiency and specific performance compared to the current state of the art, Fig. 15.

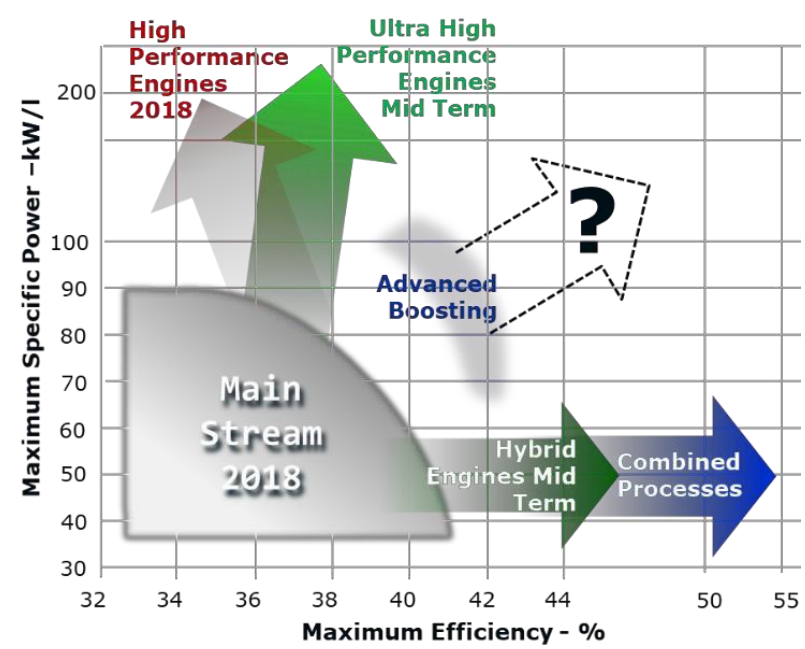

Fig. 15. Trade-off between maximum efficiency and specific power of Gasoline engines
The benchmark here are Formula 1 engines, which - not restricted by the limitations of series solutions and emission compliance - already achieve such efficiencies. If, on the other hand, one considers the boundary conditions of series engines, an increase in the compression ratio is a central topic for improving efficiency. A technically comparatively simple solution is a monovalent $\mathrm{CNG}$ engine that converts CNG's high anti-knock properties into additional efficiency improvements in addition to its chemical $\mathrm{CO}_{2}$ advantage. This is a technically ideal solution that in future will also offer the possibility of using $\mathrm{CO}_{2}$-neutral gas produced from renewable energies distributed in basically existing networks that are easy to expand. Unfortunately, this approach so far had poor customer and political resonance and thus in the past a restrained market acceptance. Hopefully CNG finally will achieve the importance it deserves.

Another much discussed approach are lean concepts. Since there are additional question marks for any form of lean operation due to the current $\mathrm{NO}_{\mathrm{x}}$ emission discussions, avoiding knocking combustion as an enabler for higher compression may be a more promising route. However, many of the conventional knocking methods are based on charge cooling and thus from the process point of view, they are a thermodynamic loss which also increases the required cooling capacity. A practically "knock-free" Gasoline engine would enable a significant paradigm shift: an increasingly adiabatic engine which transfers the wall heat losses into exhaust gas enthalpy and allows the respective utilization of this exhaust gas energy in a subsequent expansion process, Fig. 16 upper part.

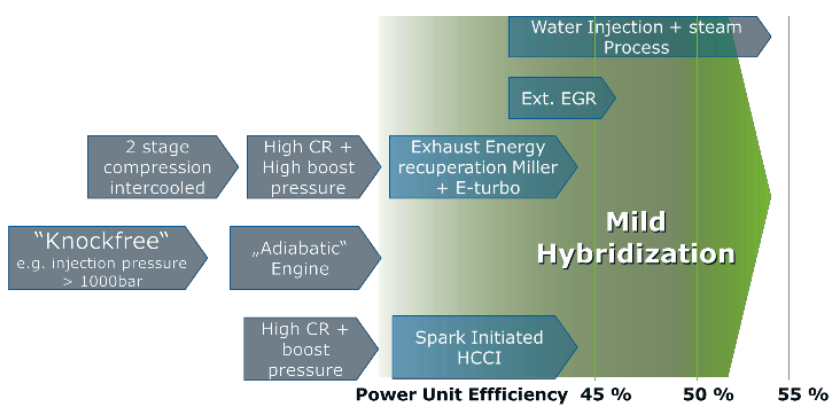

Fig. 16. Technology approaches for further increased efficiency of the Gasoline engine

Such a knock-reduced Gasoline engine would be also an ideal basis for homogeneous auto-ignition, Fig. 16 lower part. As early as 2004, AVL had demonstrated that spark ignition-triggered "auto-ignition" (AVL JCAI-Jet Controlled Auto Ignition) dramatically improves the robustness and stability of conventional HCCI technologies. However, since NOx emissions require lean exhaust aftertreatment, such a system has not been prioritized furthermore but has been switched to more emission-stable stoichiometric approaches. Another possibility for improving the knocking behavior, and also the charge dilution tolerance, is, for example, a pre-chamber spark plug, Fig. 17.

By appropriate alignment of the ignition jets, the combustion can be specifically accelerated in the direction of knock-critical areas and thus the knocking behavior can be 
improved. An advancement of $50 \%$ mass fraction burnt point of up to $8^{\circ} \mathrm{CA}$ was achieved.
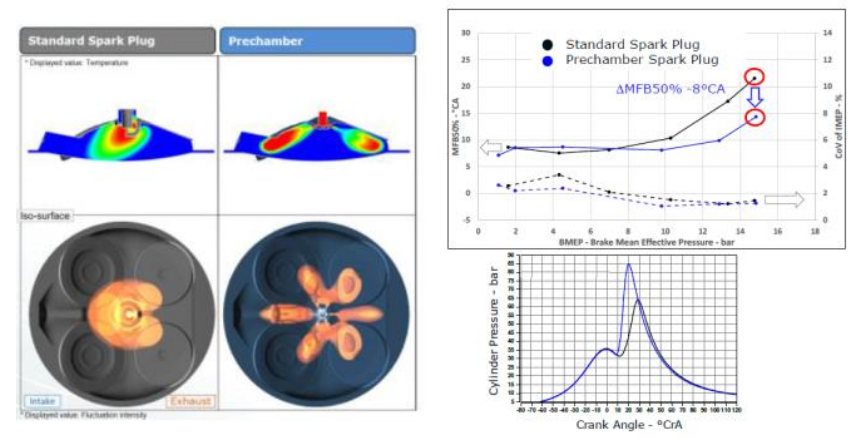

Fig. 17. Pre-chamber ignition

The most efficient measure to prevent pre-ignition and knocking, however, is the introduction of the fuel only at the end of the compression immediately before ignition, Fig. 18. The local residence time of the fuel in knockcritical areas is too low to trigger pre-ignition or knock $\Rightarrow>$ practically "knock-free" Gasoline engine.

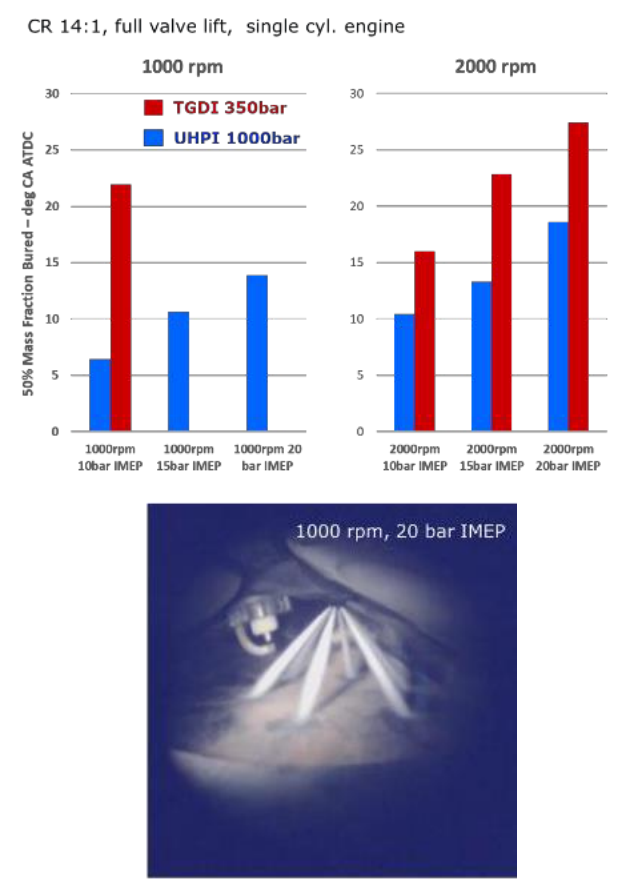

Fig. 18. "Knock-Free Engine"; Ultra High Injection Pressure

However, the very late introduction and the necessary preparation and distribution of the fuel requires extremely high injection pressures. With injection pressures $>800$ bar and an adequate system design, a sufficient mixture formation quality and homogenization can be achieved even at very late injection.

Fig. 19 shows the effect of such a knock-reduced combustion process in the engine map. With increasing the geometrical compression ratio with a standard Miller combustion system, e.g. from 12 to 14 , shifts the area of best BSFC ("sweet spot") towards lower engine loads, however, compromises the high load behavior quite significantly especially at low speeds, Fig.19 center.

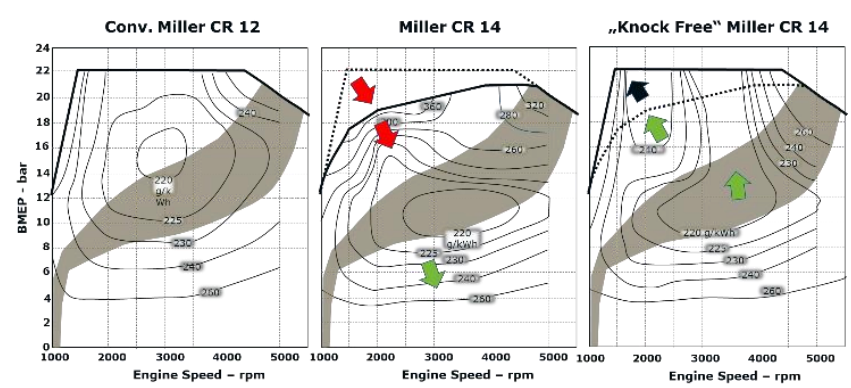

Fig. 19. Impact of a knock reduced combustion process in the engine map, no EGR

With an "ICE only" powertrain, the reduced full load torque would require a shorter drive ratio for comparable performance and thus equalize the fuel consumption benefits in the lower map range again. With a Hybrid, low-speed assistance can be provided by an electrified powertrain. Nevertheless, disadvantages would remain in the higher load range.

A knock-reduced combustion method can fully exploit the advantages of an increased compression in the lower load range, but avoids the disadvantages in the higher load range and at full load, Fig.19 right hand. Thus, such knockreduced combustion processes represent the next step in Miller concepts.

\section{Conclusions}

Regarding the improvement of real world emissions, the transition to RDE proves to be much more effective than lowering the certification values on the chassis dynamometer - by fulfilling the current RDE legislation, in real driving conditions, the RDE-induced widespread introduction of the particulate filter on the Gasoline engine and the significantly tightened specifications for cold and high load emissions result in essential pollutant reductions. It can be assumed that such vehicles will certainly fall below the imission limits even in today's imission-critical zones.

Since the extended boundary conditions of RDE legislation are no longer limited to statistically relevant driving conditions only, but can cover virtually all possible modes of operation, the short-term fulfillment of these requirements is an incredible challenge for the automotive industry.

The uncertainty of the future markets' reaction calls for the development of modular structures of both with ICE's, transmissions and electrification components.

In the long term, new potentials of the ICE can be seen with regard to both pollutant and $\mathrm{CO}_{2}$ emissions. In particular, synergy effects with electrification and the networking of powertrain control with all vehicle-relevant traffic and environmental information - "Internal Combustion Engine 4.0" - enables both further fuel consumption improvements and an emission behavior that can be described as "Zero Impact Emission". This means that future combustion engines will not have a negative impact on the pollutant imission situation. With the e-fuels theme, the combustion engine also has the potential in the medium and long term to become both $\mathrm{CO}_{2}$-neutral and pollutant-neutral. 


\section{Nomenclature}

BEV battery electric vehicle

CNG compressed natural gas

DI direct injection
ICE internal combustion engine

RDE real driving emission

SI spark ignition

\section{Bibliography}

[1] LIST, H.O. Antriebssysteme im Wandel. 39. Internationales Wiener Motorensymposium 2018.

[2] Bundesministerium für Wirtschaft und Energie; Bundesministerium für Bildung und Forschung; Plattform Industrie 4.0: Was ist Industrie 4.0?, https://www.plattformi40.de/I40/Navigation/DE/Industrie40/WasIndustrie40/wasist-industrie-40.html, 2018.

[3] KUFFERATH, A., KRÜGER, M., NABER, D. et al. Der Diesel Powertrain auf dem Weg zu einem vernachlässigbaren Beitrag bei den $\mathrm{NO}_{2}$-Immissionen in den Städten. 39. Internationales Wiener Motorensymposium 2018.

[4] COMMISSION REGULATION (EU) .../... of XXX amending Directive 2007/46/EC, Commission Regulation (EC) No.

Hubert Friedl, DEng. - AVL List GmbH.

e-mail: hubert.friedl@avl.com
Günter Fraidl, DEng. - AVL List GmbH.

e-mail: gunter.fraidl@avl.com
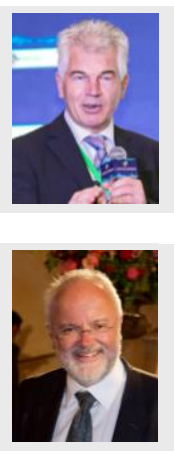

692/2008 and Commission Regulation (EU) 2017/1151 for the purpose of improving the emission type approval tests and procedures for light passenger and commercial vehicles, including those for in-service conformity and real-driving emissions and introducing devices for monitoring the consumption of fuel and electric energy (Ares (2018)1297632).

[5] SCHÖFFMANN, W., SORGER, H., WEISSBÄCK, M., Effiziente und kostenoptimierte Antriebseinheit für 48VSysteme“. MTZ. 2017, 5.

[6] PELS, T., DAVYDOV, V., ELLINGER, R. et al. 48V where to place the e-machine? 11th International MTZ Conference on Future Powertrains, 2017.

Paul Kapus, DEng. - AVL List GmbH.

e-mail: paul.kapus@avl.com 